Portland State University

PDXScholar

Systems Science Faculty Publications and

Presentations

Systems Science

$4-2012$

\title{
Freedom as a Natural Phenomenon
}

Martin Zwick

Portland State University, zwick@pdx.edu

Follow this and additional works at: https://pdxscholar.library.pdx.edu/sysc_fac

Part of the Philosophy Commons

Let us know how access to this document benefits you.

\section{Citation Details}

Zwick, M. (2012). "Freedom as a Natural Phenomenon." Presented at the 64th Annual Northwest Philosophy Conference, Oregon State University, Oct. 26-27.

This Presentation is brought to you for free and open access. It has been accepted for inclusion in Systems Science Faculty Publications and Presentations by an authorized administrator of PDXScholar. Please contact us if we can make this document more accessible: pdxscholar@pdx.edu. 


\section{NORTHWEST PHILOSOPHY CONFERENCE Oregon State University, Corvallis, Oregon, Oct 26-27, 2012}

(1) Word count: 3144 words (excl. abstract, key words, figure legend, references)

(2) Author: Martin Zwick

(3) Academic status: Professor

(4) Institutional affiliation: Systems Science Graduate Program, Portland State University

(5) Mailing address: P.O. Box 751, Portland OR 97207-0751

(6) Email address: zwick@pdx.edu

(7) Telephone number: 503-725-4987

(8) Title: Freedom As a Natural Phenomenon

(9) Abstract: 104 words

This phenomenon of "freedom" in the natural world - and indirectly the question of free will - is explored using systems-theoretic concepts that link the idea of freedom to ideas about autonomy and agency. The focus is on living systems in general, and on living systems that have cognitive subsystems more specifically. After touching on the relevance to freedom of determinism vs. randomness, the paper examines four types of freedom: (i) independence from fixed materiality, (ii) activeness that is unblocked and wholistic, (iii) internal rather than external determination, and (iv) regulation by an informational subsystem. These types of freedom are not all-or-nothing but matters of degree.

Aug 21, 2012 


\section{Table of Contents}

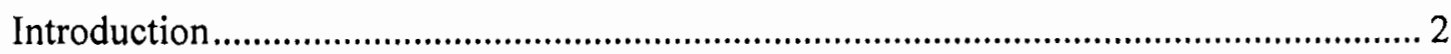

Determinism and randomness; fuzzy freedom ...................................................... 2

1. Liberation from fixed substance; available energy ................................................... 3

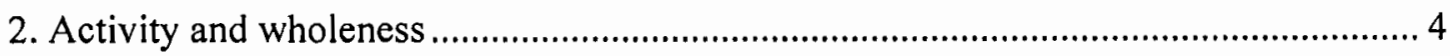

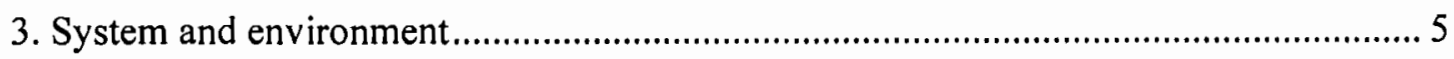

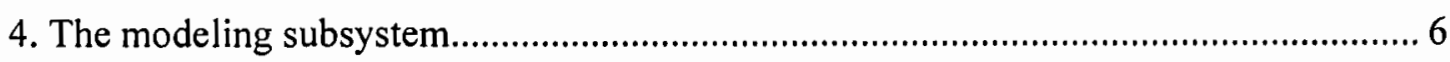

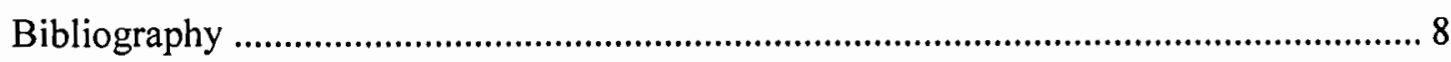

Key words: freedom, free will, determinism, agency, autonomy, autopoiesis

\section{Introduction}

This paper explores the relevance of some systems theoretic ideas to "freedom" in the natural world, and indirectly, to the subject of free will. The systems theorist Stuart Kauffman (1998) once posed the question: "what is required of a system for us to say that it "acts on its own behalf'?" This paper poses a related question: "what is required of a system for us to say that it exhibits 'freedom'?" This question is answered here by an abstract look at four types of freedom, related to autonomy and agency, that apply to living systems, or more narrowly to complex living systems having cognitive subsystems (e.g., human beings). The subject of freedom as a natural phenomenon not only has intrinsic interest, but also offers a useful broader context for discussions of free will.

As a preliminary issue, the paper discusses a "condition of possibility" for freedom, namely that the system is partially deterministic and partially random, or, alternatively, is completely deterministic but "at the edge of chaos." It then considers four different but related types of freedom. It argues that freedom inheres in:

- non-dependence on fixed matter, and availability of energy under informational control;

- activeness that is not blocked and that reflects the whole - or a central part - of the system;

- determination that is internal rather than external, and that depends on a boundary that controls interactions with the environment;

- regulation by a "modeling" (cognitive) subsystem in which information processing is hierarchical and self-referential.

In all of these, freedom is not all-or-nothing but a matter of degree.

\section{Determinism and randomness; fuzzy freedom}

Denial of freedom is based on the view that the world is governed by deterministic laws. If it is, our actions are not free. Denying determinism doesn't solve the problem. If the world is random, our actions are also not free, but in a different way. However, 
randomness allows for a variety of actions needed for freedom, so it removes an obstacle posed by determinism. And determinism causally links decision to action and action to its effects, so it removes an obstacle posed by randomness (Dennett 2003). Determinism and randomness each makes freedom impossible, but each also remedies an impossibility implied by the other.

What about a system that is partially determined and partially random? Such a system can have properties absent in systems that are wholly determined or wholly random because a whole can have properties different from - even opposite to - those of its parts. Freedom might be possible by virtue of an interaction effect between partial determinism and partial randomness. For a purely deterministic system, there is a related idea about "the edge of chaos." A deterministic system can also have chaotic attractors, where the dynamics of the system looks random but is not. Between dynamics that converges on fixed points or limit cycles and dynamics that is chaotic - analogous to the extremes of determinism and randomness - there is an intermediate regime called "the edge of chaos" (EOC), where dynamics both differs from and resembles both extremes. Langton (1991), Kauffman (1991), and others argue that all "interesting" dynamic phenomena, such as universal computation, occur in this regime. Langton also suggests that EOC systems are mathematically undecidable, so even though they are deterministic, they can have states that cannot be arrived at deterministically. Also, purely deterministic systems can have singularities at which the future is not merely unpredictable, but actually undetermined. All this does not invoke quantum mechanics, which will not be taken up here, except to note that in some of its interpretations quantum theory also harbors a mixture of determinism (in the time evolution of the wave function) and non-determinism (in the mixtures of states produced by measurement and in the uncertainty relations).

The point here is twofold: (i) we do not view the world as governed by complete determinism and (ii) partial determinism is a condition of possibility for freedom. Possibility is not actuality, but freedom as an actuality - indeed, several types of freedom - emerges in the biological realm. In the following sections, four types of freedom in this realm are discussed. These freedoms involve autonomy and agency, and are matters of degree. In set theory terminology, the set of free actions and the set of unfree actions are not taken to be "crisp" sets, where the question is whether the first set is empty. Instead, these sets are viewed as fuzzy (Zadeh 1965), which allows membership in a set to be not only 0 or 1 , but any value in between. An action can have membership in the set "free" of .1 or .5 or .9 . What is important is not the precise value but the fact that some actions are freer than others, depending on the nature of the system, on external and internal conditions, and the type of freedom under discussion.

\section{Liberation from fixed substance; available energy}

Some systems construct and maintain themselves, i.e., exhibit "autopoiesis" (selfmaking) (Maturana \& Varela 1980). They do so by informational control over a matterenergy flux through the system. Control operates (a) via a boundary that lets only some forms of matter-energy into the system; and (b) via metabolic processes that constantly reconstruct the system and, in more complex systems, reproduce it. In autopoietic systems, identity is not material but informational, so the system, although materially 
instantiated, is free from dependence upon specific and fixed materiality. For Jonas (1966) metabolism is thus the first appearance of freedom in the natural world, a freedom related to autonomy and also inextricably tied to vulnerability.

To have this type of freedom, self-construction and self-maintenance require the unceasing importing of matter-energy, hence the vulnerability. But through this importing, the system can acquire internal reservoirs of (matter and) energy. Bateson (1979) refers to this as having "collateral energy," and gives the example that a kicked dog jumps not from the force of the kick, but from its own energy sources. The dog's

jump is admittedly triggered by the external disturbance, but the point is that the dog has internal energy resources usable for active and intentional purposes. Metabolism thus brings freedom not only in liberation from fixed substance, but in provision of energy for system-directed action.

Use of this energy is governed by internal information and guided by some implicit or explicit representation of the system's "interests," in decision- and game-theoretic terms, "utility"(von Neumann \& Morgenstern 1944). Freedom, in the sense of agency, thus depends on particular relationships between matter (the physical instantiation of the system), energy (its capacity to change internally and also to affect its environment), information (governing of this capacity), and utility (the implicit or explicit goals that guide such governance). In Aristotelian terms, matter is material cause, energy is efficient cause, information is formal cause, and utility is final cause. Agency - and freedom - require a particular harmony among these four causes.

\section{Activity and wholeness}

Human action is often conceived of as driven by desire, with freedom implying the absence of obstacles (either internal or external) to the fulfillment of desire. Bennett's (1966) "systematics" speaks of desire and what opposes it in the general terms of "active" and "passive" forces. In Newtonian terms, these forces are action and reaction; in Hegelian (actually Fichtean) terms, thesis and antithesis; in Peircian (1991) terms, firstness and secondness. Two forces locked in opposition leave no room for freedom, but an added third force may allow such a possibility. Systematics defines the "triad" as including these forces: active (affirming), passive (denying, receptive), and neutralizing (reconciling, mediating), labeled 1,2, and 3, respectively. Bennett considers the six permutations, $123,132,213,231,312,321$, where 123 roughly means 1 is linked to 2 which is linked to 3 , and gives each triad a different meaning. He interprets 321 as "freedom" in the following sense: the reconciling factor (3) neutralizes the denying factor (2) in advance, removing it as an obstacle, leaving the active force (1) free to manifest without opposition. Freedom in this sense is unblocked activeness.

1,2 , and 3 could be correlated with energy, matter, and information, respectively, and 321 exemplified by metabolism, where information (enzymes) acts on matter (molecules of food) to release energy (ATP), then available for cellular processes. Other examples can be given where information (3) triggers a system that is poised to act by removing that which blocks action (2), allowing the system to actively use its available energy (1). 
Freedom requires not only unblocked activeness, but also wholeness. To be maximally free, action must reflect the whole of the system. If it arises from only part of the system, the rest of the system is passively subject to the consequences of the action and is unfree. This is relevant to free will because there are reasons to doubt that the psyche has enduring unity. Our decisions and actions are made by parts that claim to represent the whole. Each part, governed by its own utility, disregards negative externalities to other parts. If the system is united, however, multiple utilities are aggregated into some unitary utility, or an order of priority is established, or one utility is selected as the objective with the others only providing constraints on the optimization. Under these conditions, agency is more free because it is more whole. But no system is a totally integrated whole, so what is needed is only that some central aspect of the system be unitary.

Freedom requires not only unity but multiplicity, namely multiple options available to a unitary decision maker. Ashby's Law of Requisite Variety (1956) illustrates this idea by describing a necessary condition for successful regulation against disturbances from the environment. The law states that successful regulation requires a regulator that chooses deterministically an effective response for any particular disturbance. This illustrates the above assertion that determinism is required for freedom because decisions need to reliably produce actions that have reliable consequences. But Ashby's Law applies only to certain regulators. Successful regulation needn't always require deterministic action: in zero sum games without saddle points, optimal action is a mixed maximin strategy in which actions are selected randomly (but are governed by fixed probabilities).

\section{System and environment}

The environment delimits the system, imposes constraints, and presents disturbances. The impacts of the environment depend on the degree to which the system is open or closed. Closedness can shield the system from external disorder that threatens its internal order and from external order that diminishes its autonomy. Openness can protect the system from internal disorder produced entropically and internal order that reduces necessary variety. But everything depends on when, to what degree, and towards what is the system open or closed. Control of the boundary - a feature of autopoiesis - is a condition of possibility for viability and freedom.

There are two views of how systems are constituted: an open systems symmetric view in which a system is both internal structure and external function, and a closed systems asymmetric view in which structure is what the system is (internally) and function is what it does (externally). For complex systems, the asymmetric view is more appropriate. For a system to be free, internal determination must be greater than external determination. Freedom, in this sense, means being causa sui, the cause of oneself. While there is no escape from environmental constraint, disturbance, and dependence, freedom means selfdetermination, not other-determination, autonomy, not heteronomy. But autonomy is a matter of degree.

This is the deep significance of autopoiesis. In so far as the system is governed by internal information, it is "closed under causal entailment" (Rosen 1991). It is the main cause of itself - or at least of its core identity. This does not mean the system is immune to external influences, but even when internal specifications for self-construction are 
influenced by external conditions, how these conditions modify the specifications is itself (at least partially) internally specified.

Moreover the system can affect which environment it is in. Some environments impose more constraints or more benign constraints than others. Freedom is not the possibility of escaping constraint altogether, but participating in determining which constraints the system will come under. For example, locomotion allows animals some choice of environment; this freedom in space is a major evolutionary advance. In more complex animals that have "modeling subsystems," systems can gain freedom also in time, as it were, by extending the present moment through modeling to encompass past and future.

A system can satisfy external constraints before they drastically limit freedom of action. A horse tied to a moving cart gains freedom by running with the cart and avoids being dragged by it. Beyond such accommodation to environmental demand, there is the possibility also of modifying the environment to suit the system. Freedom from determination by the environment is thus achievable in multiple ways: by selecting one's environment, by accepting it or adapting to it, or by changing it.

Freedom means reducing control by the environment that is not only direct, via external limitation or compulsion, but also indirect. The environment (either other systems that it contains or the environment as a suprasystem) colonizes the system by establishing "beachheads" within it. From one perspective, this is not as restrictive as direct external control, since foreign influences can in principle come under internal control. But from another perspective, it is potentially more harmful, because foreign implants can escape detection. In its more benign impacts, the environment simply causes the system to adapt or co-determines its "phenotype," without undermining the system's organizing principle. But for its less benign impacts, the word "colonization" is apt: the system is alienated from its nature and acts on behalf of the environment rather than itself.

\section{The modeling subsystem}

Freedom from external control is necessary but not sufficient, since a system can also be controlled by internal forces. A second liberation is needed from internal control; more precisely, from internal aspects not deriving from some internal "essence" or organizing principle. Even essence might be transcended - one could call this a third liberation - but a shift of locus of control from the outside to an inner center is already a major achievement.

In complex adaptive systems, more particularly higher organisms, the informational domain includes a "modeling subsystem" (i.e., the nervous system, perhaps also the endocrine and immune systems) that models and controls the system and its interaction with the environment, and - reflexively - even models itself. Through this subsystem, systems acquire the capacity for rationality. Some views of free will ascribe freedom to this capacity, arguing that rational action is different from causal determinism. Related to this is the fact that decision theory and game theory describe rational action as dependent on utility and the suitability of means to ends. This is a final cause explanation that may seem to differ from the efficient cause explanation that is normative for physics, reflected in its use of differential equations. But differential equations actually implicitly include 
also final causes, namely the attractors that the dynamics lead to (that is why they are called attractors), and game theory can also employ differential equations, in which action depends on differences of utility. One might object that utility is not a category for physics, where causes are expressed in terms of energy, but utility is an emergent that can be adequately expressed in the language of physics. So agency and causality only appear to be radically different, which may suggest that they are different representations of a single reality.

Since the modeling subsystem is central to the system, the question of the system's freedom becomes the question of the modeling subsystem's freedom. An absence of freedom is suggested by the automatism of many of its functions. To the extent that the modeling subsystem is on auto-pilot and the reactions triggered in it are automatic and involuntary, this subsystem is unfree in being determined. To the extent that reactions do not reflect a central and enduring principle, but are contingent upon highly variable internal or external factors, this subsystem is unfree in being random. But to the extent that automatic functioning is partially determined and partially random, it has the possibility of creativity and, in a certain sense, freedom. (In any case, it is free at least in the sense that determination is internal.)

Mental activity rises above automatism when it generates subjective experience of sensations, emotions, and thoughts, here called "sensitivity," a capacity unavailable to robots or zombies. Sensitivity in turn is transcended in "consciousness," in which experience is associated with an organized and unified "self." This view, taken from Bennett (1961), proposes three levels of mental function - automatic, sensitive, and conscious - where above a level of hidden activity, there is awareness of external and internal information inputs, and above that a sense of "I" associated with this experience and the possibility of directing attention. Consciousness expands sensitivity by lowering the threshold between it and what lies beneath it; this increases the possibility of freedom by allowing impulses to be vetoed, opening up additional choices of action, and enabling deliberation.

The partial independence of consciousness from sensitivity is like a transmission disengaged by a clutch. In this decoupling, impressions are brought into view and separated to some extent from the internal or external action that they normally activate. If this energy of sensitivity is not too strong, it need not lead to habitual action or to the completion of action already incipient; the system is thus freer. If awareness is fast enough, what normally discharges impulsively can even be diverted into other channels, augmenting available energy. But such alchemy is rare; normally, sensitivity and consciousness are stuck together, without a gap that might allow freedom from impulse or awareness of factors distant from immediate impressions. Consciousness needs to be pried away from sensitivity also to prevent the complete capture of attention by either external or internal impressions. A condition of possibility of freedom for the modeling subsystem is mobility of attention.

The modeling subsystem not only models the system's interaction with the environment and the (rest of the) system, but also itself. Self-reference has already been noted as a source of freedom in autopoiesis and agency. The modeling subsystem offers another 
kind of freedom through self-reference: in the fuller perception of the energies operating within it, the subsystem, and the system as a whole, is freer. Famously, self-reference generates paradox. In Gödel's undecidability theorem, self-reference allows the existence of a well-formed formula that is meaningful but undecidable; moreover this formula, despite being undecidable, is true. Decidability in formal systems corresponds to causal determinism in physical systems. It is conceivable that self-reference allows the operation of the mind to be causa sui (Zwick 2007) - in some sense and to some degree. This is the highest form of freedom imagined in this paper.

\section{Bibliography}

Ashby, W. Ross (1956). An Introduction to Cybernetics. London: Chapman \& Hall LTD.

Bateson, Gregory (1979). Mind and Nature: A Necessary Unity. New York: Bantam Books.

Bennett, John G. (1961). The Dramatic Universe: Vol. Two: The Foundations of Moral Philosophy. London: Hodder \& Stoughton.

Bennett, John G. (1966). The Dramatic Universe: Vol. Three: Man and His Nature. London: Hodder \& Stoughton.

Dennett, Daniel (2003). Freedom Evolves. Viking: New York.

Jonas, Hans (1966). The Phenomenon of Life: Towards a Philosophical Biology. Evanston: Northwestern University Press.

Kauffman, Stuart and Johnsen, Sonke (1991). "Coevolution to the Edge of Chaos: Coupled Fitness Landscapes, Poised States, and Co-Evolutionary Avalanches." In Artificial Life II. SFI Studies in the Sciences of Complexity, vol. X, ed. by C.G. Langton, C. Taylor, J.D. Farmer, \& S. Rasmussen, Redwood City: AddisonWesley.

Kauffman, Stuart (1998). Talk at International Conference on Complex Systems, Boston.

Langton, C. (1991). "Life at the Edge of Chaos." In Artificial Life II. SFI Studies in the Sciences of Complexity.

von Neumann, John, \& Morgenstern, Oskar (1944). Theory of Games and Economic Behavior. New York: Wiley.

Peirce, Charles S. (1991). Peirce on Signs: Writings on Semiotic. James Hoopes, ed., Chapel Hill: University of North Carolina Press.

Rosen, Robert (1991). Life Itself: A Comprehensive Inquiry into the Nature, Origin, and Fabrication of Life. New York: Columbia University Press.

Toulmin, Stephen (1982). The Return to Cosmology. Berkeley: University of California Press.

Zadeh L.A. (1965). "Fuzzy Sets." Information and Control 8, 338-353.

Zwick, Martin (2007) "Spinoza and Gödel: Causa Sui and Undecidable Truth." North American Spinoza Society Monograph 13, pp. 46-52. 